\title{
Actitudes Explícitas e Implícitas hacia los Hombres Homosexuales en una Muestra de Estudiantes Universitarios en Chile
}

\author{
Explicit and Implicit Attitudes toward Gay Men \\ in a University Sample in Chile
}

\author{
Manuel Cárdenas y Jaime Barrientos \\ Universidad Católica del Norte
}

\begin{abstract}
Se reportan los resultados de un estudio sobre las actitudes implícitas de jóvenes universitarios chilenos hacia los hombres homosexuales $(N=117)$, obtenidas por medio del Implicit Association Test (IAT). Se comparan las actitudes implícitas de hombres y mujeres con las obtenidas por medio de una medida explícita, la Escala de Actitudes hacia Hombres Gay (ATG). Los hombres muestran actitudes explícitas más desfavorables hacia los homosexuales que las mujeres. Las diferencias desaparecen cuando se mide la actitud implícita, mostrando que hombres y mujeres tienen una actitud implícita negativa hacia los hombres homosexuales, lo que indicaría que la aparente disminución del prejuicio que puede constatarse en auto-reportes, o asociada a ciertos grupos, podría encubrir el hecho que las actitudes automáticas pudieran no haber cambiado.
\end{abstract}

Palabras clave: actitudes implicitas, actitudes explícitas, homofobia.

\begin{abstract}
The article reports Chilean college students' implicit attitudes toward homosexual men $(N=117)$, measured through the Implicit Association Test (IAT). The study compared young men and women implicit assessments with those obtained with an explicit measure, the Attitude toward Gay Men (ATG) Scale. Men showed more unfavorable implicit attitudes toward homosexuals than women. However, these differences were not observed when measuring implicit attitudes, showing that both men and women had negative implicit attitude toward homosexual men. These results suggest that the apparent decrease in prejudice revealed in self-reports measures, or associated with certain demographic groups, could conceal the fact that automatic attitudes might not have changed.
\end{abstract}

Keywords: implicit attitudes, explicit attitudes, homophobia.

Antecedentes

Las actitudes son evaluaciones, ya sean favorables o desfavorables, que realizan las personas sobre ciertos objetos, personas o grupos del entorno social. Tradicionalmente, se las ha considerado desde el punto de vista explícito, es decir, se refieren al auto-reporte que una persona realiza sobre sus actitudes o a la manifestación directa y explícita que una persona hace sobre un determinado objeto,

Manuel Cárdenas, Escuela de Psicología, Universidad Católica del Norte, Antofagasta, Chile.

Jaime Barrientos, Escuela de Psicología, Universidad Católica del Norte, Antofagasta, Chile.

La correspondencia relativa a este artículo debe ser dirigida a Jaime Barrientos Delgado, Escuela de Psicología, Universidad Católica del Norte, Avenida Angamos 0610, Antofagasta, Chile. E-mail: jbarrien@ucn.cl persona o grupo. Los instrumentos tradicionales para evaluar estas actitudes han sido cuestionarios y escalas graduadas. Sin embargo, estos instrumentos de evaluación han sido criticados debido al gran número de limitaciones detectadas en ellos, tales como la diferente capacidad de las personas de darse cuenta o ser consciente de sus propias actitudes y estados internos, la deseabilidad social de las respuestas, la presentación positiva que intentan hacer de sí mismos (ya sea ante otros o ante sí mismos en vistas a preservar una auto-imagen positiva) o los intentos de corregir los juicios en aquellas escalas reactivas al momento de detectar cuál es la variable que se está midiendo (Briñol, De la Corte \& Becerra, 2001; Petty \& Wegener, 1998).

Como consecuencia de las limitaciones de los procedimientos de auto-reporte, se han diseñado instrumentos no reactivos o indirectos que permitan el acceso a los estados internos y actitudes de las 
personas, sin que se les consulte directamente sobre estos. Estas medidas implícitas permitirían una evaluación más rápida, menos consciente, menos controlable y más difícil de corregir o ajustar a las expectativas (Greenwald \& Banaji, 1995). Los dos instrumentos más conocidos y utilizados en este ámbito son la Tarea de Evaluación Automática (Fazio \& Olson, 2003; Fazio, Sanbonmatsu, Powell \& Kardes, 1986) y el Test de Asociación Implícita (Greenwald, McGhee \& Schwartz, 1998; Greenwald \& Nosek, 2001). El supuesto que está a la base de los instrumentos indirectos de medida es que buena parte de la vida de las personas está condicionada por procesos mentales que son activados de forma automática (Devine, 1989) y que cursan sin conciencia para los individuos (Bargh, 1999), delimitando fuertemente los grados de libertad de los mismos, tanto para responder a su entorno como para modular sus juicios y decisiones. En este sentido, estaríamos frente a la presencia de actitudes que son activadas de forma automática y que son desconocidas para los individuos, aunque no por ello dejan de influir en las respuestas de una persona hacia un determinado objeto, persona o grupo (Greenwald \& Banaji, 1995).

En esta investigación se usó el Test de Asociación Implícita (IAT, por su nombre en inglés Implicit Association Test). Este instrumento mide los tiempos de reacción en tareas de clasificación de palabras a determinados estímulos, midiendo la fuerza asociativa de dos conceptos y dos atributos. El supuesto a la base de este instrumento es que resulta más fácil y rápida la respuesta conductual cuando dos conceptos fuertemente asociados comparten una misma categoría. Por tanto, la latencia de respuesta permite medir la fuerza relativa de la asociación entre conceptos, lo que permite inferir las actitudes hacia los mismos (Greenwald et al., 1998; Greenwald \& Nosek, 2001; Nosek, Greenwald \& Banaji, 2005).

En Chile, diversos estudios sobre actitudes explícitas hacia diversos temas han sido efectuados en los últimos años (Cárdenas, 2006; González et al., 2005; Herrera, Mathiesen, Morales, Proust \& Vergara, 2006; Merino, 2006; Mladinic, Saiz, Díaz, Ortega \& Oyarce, 1998), así como, también, diversos estudios específicos sobre sexualidad y género (Diekman, Eagly, Mladinic \& Ferrerira, 2005; Gavilán, 2005; Godoy, 2007; González, Molina, Montero, Martínez \& Leyton, 2007; Matus, 2005; Sharim, 2005; Valenzuela \& Casas, 2007). Estudios generales sobre homosexualidad y su relación con diversos aspectos demográficos, sociales y éticos han sido realizados en diversos países, incluido Chile (Montoya, 2006; Montoya-Montoya, Risco, Rodríguez, Valdebenito \& Lolas, 2006). Sin embargo, las investigaciones sobre homofobia son menos frecuentes y la mayoría han sido realizadas en México y Brasil (Altair \& Caetano, 2007; Carrara \& Vianna, 2006; Ortiz-Hernández, 2004; Ortiz-Hernández \& García, 2005a, 2005b; Sevilla \& Álvarez, 2006). Finalmente, los estudios efectuados en la región, así como en Chile, y que utilizan medidas de homofobia lo hacen sin indicar las propiedades métricas de los instrumentos (Galdames \& Aron, 2007), refiriéndose principalmente a medidas de auto-reporte (Barrientos \& Páez, 2000; Blümel, Binfa, Cataldo, Carrasco, Izaguirre \& Sarrá, 2004) y asociadas a un ítem único que pretende medir la homofobia (Ideas, 1997, 2001, 2003; Inglehart \& Welzel, 2005). Recientemente, se han realizado en Chile estudios con medidas explícitas de actitudes hacia la homosexualidad con sus respectivos procesos de adaptación y validación (Cárdenas \& Barrientos, 2008; Nierman, Thompson, Bryan \& Mahaffey, 2007). En el estudio de Cárdenas y Barrientos (2008), las actitudes hacia gay y lesbianas son afectadas por el sexo del respondiente. Las mujeres fueron más tolerantes hacia los homosexuales (gay y lesbianas) que los hombres. En el mismo estudio se constató que las personas más intolerantes son aquellas más religiosas, tal como ha sido observado en otros estudios (Gentry, 1987; Herek, 1988; Morrison \& Morrison, 2002). En el estudio de Nierman et al. (2007), en el que se utiliza la Escala de Actitudes hacia Lesbianas y Hombres Gay (ATLG) para comparar Estados Unidos y Chile, se constató la importancia de los roles de género y su asociación con la homofobia. Así, aquellas personas que transgreden los roles de género son peor evaluadas que aquellas que no.

Respecto de la homofobia en Chile, diversos estudios indican que aún hay actitudes hostiles hacia los homosexuales, lo que puede traducirse en altos niveles de discriminación y victimización hacia las personas homosexuales. Por ejemplo, según la encuesta de Comportamiento Sexual del Cono Sur, solo un $5 \%$ de la población está de acuerdo con que hombres o mujeres tengan relaciones sexuales con personas de su mismo sexo (Barrientos \& Páez, 2000). El estudio de la Corporación Chilena de Prevención del SIDA (1997) indicó que un 38,1\% ha sufrido algún tipo de violencia a causa de su orientación sexual en los últimos cinco años. El último informe del Movimiento de Integración y Liberación Homosexual ([MOVILH], 2007) seña- 
la que en el año 2007 han aumentado un 16\% las denuncias sobre hechos homofóbicos respecto del año 2006, lo que se traduce en 57 casos (asesinatos, agresiones civiles, atropellos policiales, discriminación laboral y educacional, entre otros). Por su parte, la Encuesta de Cohesión Social en América Latina (MOVILH, 2007) indicó que al 27\% de los encuestados le molestaría mucho o algo que su hijo/a tuviera un amigo/a homosexual. Y, según el mismo estudio, Chile, comparado a otros países de la región, ocupa el cuarto lugar de menor a mayor intolerancia. La homofobia es mayor en regiones, en hombres y en menores niveles educativos. Por su parte, la Quinta Encuesta Nacional de la Juventud (Instituto Nacional de la Juventud, 2007) indica que el $21,1 \%$ de los jóvenes considera a gay y lesbianas como vecinos indeseados, ocupando el cuarto lugar de los más indeseados (neonazis, drogadictos, alcohólicos y skinheads). Asimismo, muestra que los hombres $(24,6 \%)$ discriminan más que las mujeres $(17,4 \%)$.

Otros antecedentes disponibles, más sistemáticos y periódicos, han sido obtenidos por la Encuesta de Tolerancia y No Discriminación (Ideas, 2003) y la World Values Survey ([WVS], 2005). La primera encuesta indica que más del $50 \%$ de la población podría ser tildada de homofóbica (entendida esta como una forma de prejuicio personal o institucional hacia los homosexuales, que se manifiesta en temor o rechazo a la cercanía física con personas homosexuales), en tanto, considera que la homosexualidad debe ser un importante tema de investigación médica que permita evitar que en el futuro sigan naciendo homosexuales. En el informe 2001 de la misma encuesta, un $45,2 \%$ opina que la homosexualidad debería ser prohibida, pues va contra la naturaleza humana (Ideas, 2001) y el $43 \%$ afirma que a los homosexuales no se les debe permitir ser profesores en un colegio (Ideas, 2003). Por otra parte, los datos recogidos por la WVS aportan más información sobre el tema en cuestión. Según ellos, hay un cambio positivo en las actitudes hacia la homosexualidad, aunque persiste la homofobia: de una media de 2,91 en el año 1990 se ha pasado a una de 3,98 en el año 2000 (la puntuación de la escala oscilaba entre 1 y 10 , expresando las puntuaciones superiores actitudes más favorables). Este mismo estudio indica que son más tolerantes las mujeres y los más jóvenes. Respecto a la religión, los protestantes son los más intolerantes y los católicos los más tolerantes. Considerando el nivel socioeconómico (NSE), aquellas personas de NSE bajo son las más intolerantes y las de NSE alto, las más tolerantes. Sin embargo, estos últimos dos estudios, si bien aportan información relevante, son sondeos de opinión muy amplios (en los que las preguntas acerca de la homosexualidad se limitan a una, como en el caso de la WVS, o a dos o tres ítems, como es el caso de los estudios de la Fundación Ideas) o son escalas que no aportan ningún indicador sobre la validez o la confiabilidad de la medida.

Finalmente, el instrumento escogido para este estudio permitirá constatar si los ideales democráticos en boga en el contexto chileno en diversos espacios sociales y la alta valoración de las ideas igualitarias que les son concomitantes (Díaz \& Druker, 2007; Gómez, 2007; Vega, Bedregal, Jadue \& Delgado, 2003) han contribuido a contener las expresiones abiertas de homofobia antes descritas. Además, permitirá constatar si ha disminuido la homofobia en el país, tal como lo exhorta la declaración de Santiago del año 2000 (Programa Conjunto de las Naciones Unidas sobre el VIH/Sida [ONUSIDA], 2006), o si se trata más bien de una adecuación del prejuicio a estos discursos por la vía de adaptar sus formas a unas más sutiles y encubiertas o que, incluso, cursan sin conciencia por parte de los individuos que lo ejercen.

De este modo, la primera hipótesis predice que hay diferencias significativas entre hombres y mujeres en la medida explícita de prejuicio. Es decir, a nivel manifiesto, los hombres y las mujeres difieren en su apreciación respecto de los homosexuales. La segunda hipótesis plantea que las diferencias entre hombres y mujeres desaparecen en la medida de actitudes implícitas, es decir, ambos grupos poseen actitudes desfavorables hacia los hombres homosexuales a nivel implícito.

\section{Método}

\section{Muestra}

Se usó una muestra no probabilística intencional, ya que fue seleccionado un grupo de fácil acceso, lo que hace imposible la estimación del error muestral $\mathrm{y}$, por tanto, es una muestra cuyas características hacen que los resultados contengan un sesgo importante. Este tipo de muestras es muy común en el trabajo con IAT, dadas las dificultades de reclutamiento que implica que las personas deban ser conducidas 
a un lugar especialmente habilitado donde puedan contestar la prueba frente a un ordenador.

La muestra quedó conformada por 117 participantes, 73 hombres $(62,4 \%)$ y 44 mujeres $(37,6 \%)$, cuyas edades fluctuaban entre 17 y 25 años $(M=$ $18,64$ años, $D S=1,03)$. Todos los participantes de la muestra eran estudiantes de primer o segundo año de la carrera de plan común de Ingeniería de la Universidad Católica del Norte.

\section{Instrumento}

El instrumento utilizado quedó compuesto por tres secciones diferentes. La primera de ellas incluye variables sociodemográficas (sexo y edad), las que fueron utilizadas para generar los grupos de comparación.

La segunda sección quedó compuesta por una adaptación de la Escala ATLG (Herek, 1984), la que fue utilizada como medida explícita de las actitudes. Se usó la versión adaptada y validada en una muestra nacional (Cárdenas \& Barrientos, 2008). Esta escala consta, originalmente, de 20 ítems cuyas respuestas toman la forma de una escala Likert, con opciones que van desde 1 (completamente en desacuerdo) hasta 5 (completamente de acuerdo). De esta escala se usaron solo diez reactivos (aquellos referidos a las actitudes hacia los hombres homosexuales [ATG]). La consistencia interna de esta escala fue 0,82 (coeficiente alfa de Cronbach).

El tercer bloque consistió en una adaptación de la tarea de IAT a la medida de la homofobia, la que implicó parear los conceptos diana homosexual y heterosexual con los conceptos atributo bueno y malo. Las palabras asociadas a los conceptos diana consistieron en términos relacionados con los conceptos homosexual (homo, bisexual y gay) y heterosexual (masculino, macho y hombre).

Cinco jueces expertos en el área (tres hombres y dos mujeres) evaluaron los términos. Estos propusieron aquellos asociadas a los conceptos diana, seleccionándose aquellos que fueron comunes a los cinco jueces, resguardando que dichos listados cumplieran con el criterio de ausencia de valencia para los conceptos diana, de modo de salvaguardar que los términos fueran representativos de cada categoría.

Para los conceptos atributo se utilizó un listado de rasgos para los términos bueno (placer, fortaleza y bienestar) y malo (dolor, debilidad y malestar), pero cuidando de resguardar los criterios de exclusividad y similitud de valencia (estos términos son parte de una lista internacionalmente aceptada que aludirían interculturalmente a dichos conceptos atributo) (Greenwald \& Nosek, 2001; Nosek, Greenwald \& Banaji, 2007).

Se realizó una serie de ensayos para capacitar a los participantes en la clasificación de palabras simples (e.g., heterosexual y homosexual) y combinadas (homosexual y bueno o heterosexual y malo). Se controló el orden de presentación de las combinaciones y se contrabalanceó la situación (el concepto heterosexual aparecía en algunos ensayos combinado con los atributos bueno y en otros, junto a malo. Además, aparecía la mitad de las veces en el lado izquierdo de la pantalla y la otra mitad al lado derecho de la misma).

Las actitudes implícitas fueron medidas a través de los tiempos de reacción que emplearon los participantes en la tarea de clasificación de palabras. Además, las respuestas demasiado rápidas (por debajo de 300 milisegundos) y aquellas demasiado lentas (por encima de 3.000 milisegundos) no se contemplaron en el análisis, puesto que se interpretan como respuestas de tipo anticipatorio, en el primer caso, y de tipo controlado, en el segundo. Además, los tiempos de reacción fueron transformados, utilizando el algoritmo desarrollado por Greenwald, Nosek y Banaji (2003) ${ }^{1}$.

Respecto a la validez del IAT, existen diversos estudios que muestran que sus propiedades métricas resultan suficientemente robustas (Greenwald \& Nosek, 2001; Greenwald et al., 2003; Maison, Greenwald \& Bruin, 2004; Nosek et al., 2005, 2007; Poehlman, Uhlmann, Greenwald \& Banaji, 2006), estableciendo que este instrumento puede detectar diferencias en: (a) asociaciones casi universales en la población (e.g., preferencia por las flores frente a los insectos), (b) asociaciones entre distintas poblaciones de individuos (e.g., entre americanos coreanos y

\footnotetext{
Este algoritmo es calculado sobre la base de una serie de recomendaciones de los autores: (a) suprimir los juicios que superen los diez mil milisegundos; (b) eliminar del análisis a los individuos cuyas respuestas sean inferiores a 300 milisegundos en más de un $10 \%$ de sus respuestas; (c) cálculo de la desviación estándar global para todos los ensayos en las etapas 3, 4, 6 y 7; (d) cálculo de la media latente para todos los participantes en las etapas 3, 4, 6 y 7; (e) cálculo de dos medias diferentes (media ${ }_{\text {etapa } 6}$ - media ${ }_{\text {etapa } 3}$ ) y (media $a_{\text {etapa } 7}$ media $_{\text {etapa } 4}$ ); (f) dividir cada una de las medias anteriores por su desviación estándar global y (g) la puntuación D será igual al promedio de los dos cocientes resultantes (Greenwald et al., 2003). El algoritmo se encuentra disponible como sintaxis SPSS en la página del primero de los autores.
} 
americanos japoneses, en actitudes asociadas con sus respectivas etnias) y (c) asociaciones que muestran vestigios cognitivos de una cultura racista (e.g., actitudes más favorables hacia las personas de raza blanca que hacia las personas de color). En particular, la medida del efecto IAT no se ve influenciada por la asignación de un concepto atributo determinado (e.g., bueno) a la mano izquierda o derecha, por variaciones en el intervalo de tiempo transcurrido entre sucesivos estímulos (entre 150 y $750 \mathrm{~ms}$ ) ni por el número de estímulos elegidos para representar cada categoría (i.e., 5 ó 25 estímulos). Los principales resultados con respecto a los distintos aspectos métricos de las medidas del IAT en estudios internacionales (confiabilidad test-retest, validez de constructo, validez convergente y validez predictiva) se detallan en una serie de artículos ya publicados (Greenwald \& Nosek, 2001; Nosek et al., 2007; Poehlman et al., 2006). Cabe señalar que, si bien es cierto que las propiedades del IAT se han evaluado en países diferentes del nuestro y sobre conceptos distintos a la homosexualidad masculina, lo que se ha probado y validado es el procedimiento general y no una medida específica, lo que transforma al IAT en una sólida herramienta para el análisis de todo tipo de asociaciones implícitas.

\section{Procedimiento}

Los estudiantes participaron voluntariamente, por medio de un consentimiento verbal, explicándoseles detalladamente los objetivos y los aspectos generales de la investigación. En este aspecto se siguieron las pautas éticas internacionales para la investigación biomédica con seres humanos del Council for International Organizations of Medical Sciences (2002), referidas a la investigación en la que participan personas vulnerables, las pautas de ONUSIDA (2006), así como los lineamientos respecto a la confidencialidad sobre la homosexualidad sugeridos para contextos clínicos en Chile (Montoya-Montoya et al., 2006). Adicionalmente, se siguieron los criterios éticos de la Comisión Nacional de Investigación Científica y Tecnológica, sobre todo el referido a la confidencialidad de la información producida.

Los estudiantes realizaron primero la tarea del IAT, para lo cual se habilitaron salas con computadores que contenían el software y la tarea del IAT preparada para la ocasión. Una vez terminada la tarea del IAT, los participantes procedían a rellenar el cuestionario de lápiz y papel que contenía la medida explícita y las variables sociodemográficas. El estudio fue realizado durante el mes de diciembre de 2007, en el horario de clases de los participantes.

\section{Resultados}

Las hipótesis de este estudio fueron confirmadas en la muestra. La primera hipótesis afirmaba la existencia de diferencias entre los hombres $(M=3,25$, $D S=0,65)$ y las mujeres $(M=2,81, D S=0,80)$ en sus actitudes explícitas hacia los homosexuales. Estas diferencias son confirmadas $(t[115]=3,19, p<0,01)$. De este modo, los hombres obtienen puntuaciones significativamente más altas que las mujeres en la escala ATG que mide actitudes hacia los hombres homosexuales (Figura 1). Estas puntuaciones indican que los hombres heterosexuales de esta muestra poseen y expresan de forma explícita actitudes más desfavorables hacia los hombres homosexuales que las mujeres que participaron en el estudio.

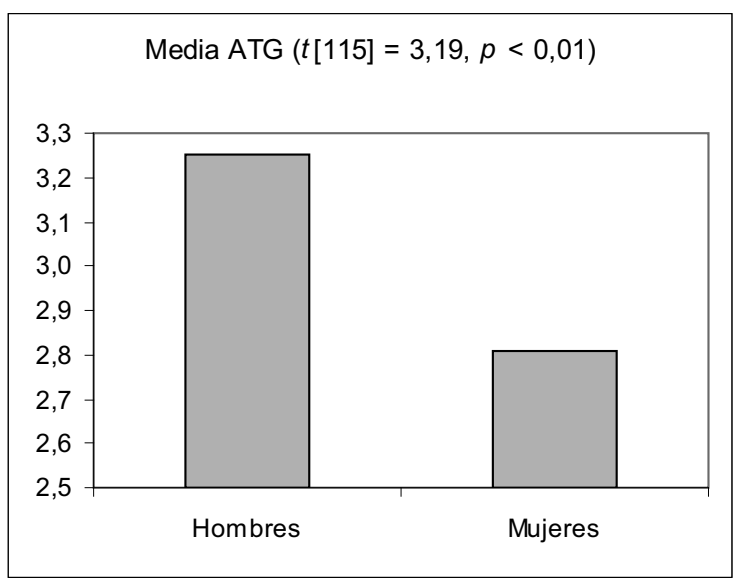

Figura 1. Comparación de medias entre hombres y mujeres en la medida explícita de actitudes hacia los homosexuales.

Respecto de la segunda hipótesis sobre la igualdad de medias entre los participantes de diferente sexo, tanto los hombres $(M=0,88, D S=0,15)$ como las mujeres $(M=0,10, D S=0,22)$ manifiestan similares niveles de prejuicio implícito hacia los hombres homosexuales (Figura 2), no encontrándose diferencias significativas entre sus puntuaciones 
$(t[115]=-0,61, p>0,05)$. Lo anterior indica que aquellas diferencias halladas a nivel manifiesto se diluyen a nivel implícito. Por tanto, aun cuando las mujeres de la muestra poseen actitudes explícitas más favorables que los hombres, a nivel automático ellas responden de la misma forma que los hombres, esto es, expresando una actitud negativa.

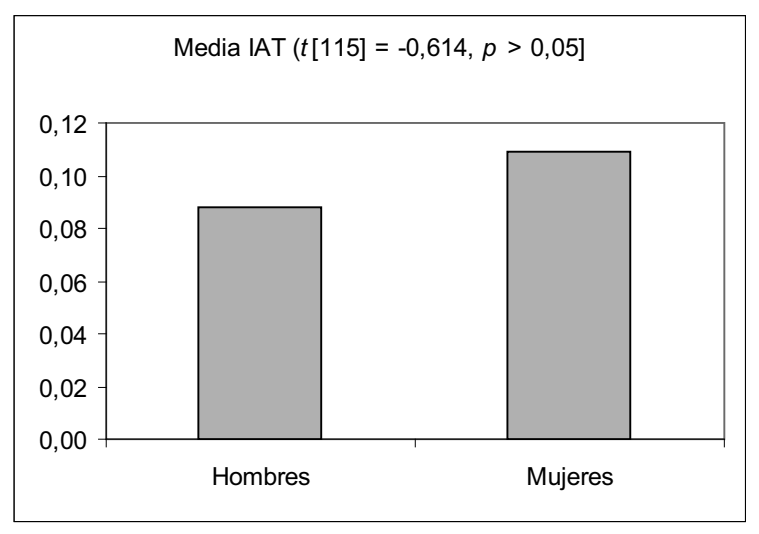

Figura 2. Comparación de medias entre hombres y mujeres en la medida implícita de actitudes hacia los homosexuales.

Finalmente, la correlación obtenida entre la medida explícita (ATG) y la implícita (IAT) fue estadísticamente no significativa para el total de la muestra $(r[117]=-0,002, p>0,05)$. Asimismo, tanto la correlación para la muestra de hombres $(r[73]=0,090, p>0,05)$ como para la de mujeres $(r[44]=-0,048 p>0,05)$ es muy próxima a cero $\mathrm{y}$ no significativa, lo que podría indicar que se trata de constructos claramente diferenciados y no relacionados entre sí. Adicionalmente, las correlaciones anteriores indicarían que una misma persona puede perfectamente expresar abiertamente actitudes de tipo igualitario, pudiendo aparecer como tolerante, pero eso no implicaría, necesariamente, que esto sea así en todos los ámbitos. Es decir, cuando el estímulo es más ambiguo y se exige del individuo una respuesta rápida y no deliberada, entonces los individuos pueden expresar actitudes negativas. Estas actitudes, incluso, podrían cursar sin conciencia y sin necesidad de cuestionar la auto-imagen positiva de la persona.

\section{Discusión}

Los resultados obtenidos muestran cómo el grupo de mujeres, que en principio aparece como menos prejuicioso (medida explícita), no muestra diferencias significativas con el grupo de hombres cuando son evaluadas sus actitudes de una manera indirecta (medida implícita). Es decir, este grupo se comporta de forma tal que muestra actitudes hacia la homosexualidad masculina significativamente más negativas en la medida implícita que en la explícita. Para los hombres, ambos grupos de actitudes no se diferencian, aunque obtienen medias significativamente más elevadas en la medida explícita que las mujeres. Lo anterior indicaría cómo han cambiado las formas de expresión del prejuicio desde modos más bien abiertos y hostiles, hacia unos más elaborados y sutiles, al menos, para el grupo de mujeres para el que los resultados obtenidos revelan niveles menores de prejuicio explícito. Además, se observa como el prejuicio puede cursar sin que las personas sean necesariamente conscientes del mismo. No se trata ya de formas más racionalizadas sino, al contrario, de formas inconscientes, implícitas o automáticas. El uso de la tecnología disponible permite acceder a los estados internos de las personas sin necesidad de preguntar directamente por ellos, evitando el proceso de racionalización y permitiendo apreciar sus respuestas automáticas ante la presencia de un estímulo relevante.

Por otra parte, del conocimiento de las actitudes no se deriva necesariamente prejuicio explícito. Tampoco las ideas sobre uno mismo o los esfuerzos que conscientemente se hacen por actuar de modo igualitario frente a los otros grupos sociales son, necesariamente, un revulsivo contra el prejuicio o la asociación de ciertos grupos a ciertos contenidos negativos. Así, los participantes de esta muestra -sin distinción de sexo y de sus actitudes explícitas- manifiestan niveles de prejuicio implícito que indican que, a nivel latente, disponen de asociaciones hacia los homosexuales que, una vez que se hacen salientes en el campo social, les imponen ciertos automatismos hacia dicho grupo. Es decir, aun cuando las personas crean ser genuinamente igualitarios en el trato con los hombres homosexuales pueden estar, en un nivel latente o inconsciente, haciendo uso de prejuicios.

En definitiva, se observa que, a nivel automático o implícito, los participantes de la muestra pueden estar activando asociaciones que los hacen vincular la homosexualidad masculina con evaluaciones negativas. Se aprecia la fuerte asociación que existe 
entre las propiedades del objeto de análisis (orientación sexual) y las dimensiones de juicio (atributos). Por tanto, simples términos asociados a la homosexualidad hacen saliente asociaciones implícitas con conceptos negativos de forma más fácil que con aquellos positivos. Lo anterior se observa en el grupo de mujeres, quienes, en la medida explícita, no muestran actitudes muy negativas, pero sí lo hacen al reaccionar de forma automática a ciertos estímulos vinculados a la homosexualidad masculina. El caso de los hombres, en cambio, es aún más llamativo ya que, al parecer, manifiestan directa y abiertamente sus actitudes negativas, lo que indicaría que las formas de expresión están lejos de verse afectadas por los cambios en el contexto y la presión de los valores igualitarios. En el futuro podrían realizarse estudios con instrumentos explícitos, pero de índole más sutil, que permitan probar los cambios en los modos de expresión a nivel manifiesto.

Estos antecedentes abren una discusión acerca de la determinación inconsciente de las acciones humanas y la necesidad de modificar, a ese nivel, estereotipos sociales que contribuyen a mantener relaciones que desfavorecen a un grupo social y que son mantenidos debido a la falta de reflexión sobre los mismos. Este patrón automático es el que permite un enraizamiento profundo de las prácticas sexistas y discriminatorias, amparado en un discurso explícito que aboga por la igualdad de género y la no discriminación por la orientación sexual de las personas, lo que permite mantener la auto-imagen positiva del individuo. La falta de reflexión y el automatismo de la conducta de las personas contribuyen a sostener relaciones desiguales y poco equitativas entre sexos y diferentes orientaciones sexuales, encubriendo el prejuicio por medio de un discurso igualitario.

Este enraizado discurso sobre la homosexualidad puede indicar, indirectamente, que la categoría género sigue siendo sumamente importante y pertinente en Chile para segmentar y jerarquizar a los grupos sociales. Es decir, cumpliría una función legitimadora que permite sostener la desigualdad y mantener el dominio de unos grupos sobre otros. En este sentido, sería interesante saber si el comportamiento es similar en el caso de las mujeres lesbianas. También, hay que observar el efecto diferencial que se obtiene al comparar a otros grupos mediante la condición de género, como es el caso de hombres y mujeres. Probablemente, los discursos públicos hayan cambiado pero, a nivel latente, se mantengan aún actitudes negativas hacia las mujeres, las cuales afloran en contextos menos regulados o que implican respuestas no deliberadas. Este efecto implicaría que sería el desvío de los roles de género y no la homosexualidad en sí misma la que sería sancionada por las personas. Esto, evidentemente, deberá ser probado en futuros estudios, los cuales tendrían que considerar las construcciones culturales del género y la sexualidad en Chile.

Adicionalmente, futuros estudios podrían centrarse en la relación existente entre estereotipos y actitudes implícitas, ya que los estereotipos no se asocian de manera directa con las actitudes explícitas (del estereotipo no se deriva necesariamente una actitud negativa), pero se desconoce su efecto sobre las actitudes implícitas. Es decir, tanto las personas prejuiciosas como aquellas no prejuiciosas conocen y pueden hacer accesible, en todo momento, el estereotipo sobre un determinado grupo. Y, si bien este estereotipo no determina la presencia de discriminación de forma explícita, este parece estar íntimamente vinculado con la aparición de asociaciones a nivel subyacente, las que podrían determinar respuestas discriminatorias sin necesidad de intervención de la voluntad de la persona, impidiendo con ello que se produzca un cuestionamiento de la identidad positiva del individuo que se supone a sí mismo igualitario.

Finalmente, otra limitación del estudio se refiere al uso de muestras intencionales de estudiantes universitarios. Los límites de la generalización de los hallazgos con este tipo de muestras está documentado, pero en Chile los estudios sobre este tópico están recién comenzando (Herek \& GonzálezRivera, 2006).

\section{Referencias}

Altair, F. \& Caetano, H. (2007). Saindo do armário e entrando em cena: juventudes, sexualidades e vulnerabilidade social. Revista Estudos Feministas, 15, 45-66.

Bargh, J. A. (1999). The cognitive monster: The case against the controllability of automatic stereotypes effects. En S. Chaiken \& Y. Trope (Eds.), Dual-process theories in social psychology (pp. 361-382). New York: Guilford Press.

Barrientos, J. \& Páez, D. (2000). Identidad homo-bisexual en Chile. En Comisión Nacional del SIDA \& Agence Nationale de Recherches sur le SIDA, Estudio nacional de comportamiento sexual. Primeros resultados (pp. 123-129). Santiago, Chile: Ministerio de Salud.

Briñol, P., De la Corte, L. \& Becerra, A. (2001). ¿Qué es persuasión? Madrid: Biblioteca Nueva.

Cárdenas C. M. (2006). «Y verás como quieren en Chile...»: un estudio sobre el prejuicio hacia los inmigrantes bolivianos por parte de los jóvenes chilenos. Última Década, 14(24), 99-124. 
Cárdenas, M. \& Barrientos, J. (2008). The Attitudes toward Lesbians and Gay Men Scale (ATLG): Adaptation and testing the reliability and validity in Chile. Journal of Sex Research, $45,140-149$.

Carrara, S. \& Vianna, A. R. B. (2006). “Tá lá o corpo estendido no chão...": a violência letal contra travestis no município do Rio de Janeiro. Physis, 16, 233-249.

Corporación Chilena de Prevención del SIDA (1997). De amores y sombras. Poblaciones y culturas homo y bisexuales en hombres de Santiago. Santiago, Chile: Autor.

Council for International Organizations of Medical Sciences (2002). Pautas éticas internacionales para la investigación biomédica en seres humanos. Geneva, Suiza: Autor.

Devine, P. (1989). Stereotypes and prejudice: Their automatic and controlled components. Journal of Personality and Social Psychology, 56, 5-18.

Díaz, T. \& Druker, S. (2007). La democratización del espacio escolar: una construcción en y para la diversidad. Estudios Pedagógicos, 33(1), 63-77.

Diekman, A., Eagly, A., Mladinic, A. \& Ferrerira, M. C. (2005). Dynamic stereotypes about women and men in Latin America and the United States. Journal of Cross-Cultural Psychology, 36, 209-226.

Fazio R. H. \& Olson, M. A. (2003). Implicit measures in social cognition research. Annual Review of Psychology, 54, 297327.

Fazio, R. H., Sanbonmatsu, D. M., Powell, M. C. \& Kardes, F. R. (1986). On the automatic activation of attitudes. Journal of Personality and Social Psychology, 50, 229-238.

Galdames, S. \& Arón, A. M. (2007). Construcción de una escala para medir creencias legitimadoras de violencia en la población infantil. Psykhe, 16(1), 15-25.

Gavilán, V. (2005). Representaciones del cuerpo e identidad de género y étnica en la población indígena del norte de Chile. Estudios Atacameños, 30, 135-148.

Gentry, C. (1987). Social distance regarding male and female homosexuals. The Journal of Social Psychology, 127, 199-208.

Godoy, L. (2007). Fenómenos migratorios y género: identidades femeninas "remodeladas". Psykhe, 16(1), 41-51.

Gómez, V. (2007). Mujeres y participación política: ¿es viable una ley de cuotas en Chile? Revista Universum, 22(1), 252-267.

González, R., Manzi, J., Cortés, F., Torres, D., De Tezanos, P., Aldunate, N., Aravena, M. T. \& Saiz, J. L. (2005). Identidad y actitudes políticas en jóvenes universitarios: el desencanto de los que no se identifican políticamente. Revista de Ciencia Política, 25(2), 65-90.

González, E., Molina, T., Montero, A., Martínez, V. \& Leyton, C. (2007). Comportamientos sexuales y diferencias de género en adolescentes usuarios de un sistema público de salud universitario. Revista Médica de Chile, 135, 1261-1269.

Greenwald, A. G. \& Banaji, M. R. (1995). Implicit social cognition: Attitudes, self-esteem, and stereotypes. Psychological Review, 102, 4-27.

Greenwald, A. G., McGee, D. E. \& Schwartz, J. L. (1998). Measuring individual differences in implicit cognition: The Implicit Association Test. Journal of Personality and Social Psychology, 74, 1469-1480.

Greenwald, A. G. \& Nosek, B. A. (2001). Health of Implicit Association Test at age 3. Zeitschrift für Experimentelle Psychologie, 48, 85-93.

Greenwald, A. G., Nosek, B. A. \& Banaji, M. R. (2003). Understanding and using the Implicit Association Test: I. As improved scoring algorithm. Journal of Personality and Social Psychology, 85, 197-216.

Herek, G. (1984). Beyond homophobia: A social psychological perspective in attitudes toward lesbians and gay men. Journal of Homosexuality, 10, 2-17.

Herek, G. (1988). Heterosexual's attitudes toward lesbians and gay men: Correlates and gender differences. The Journal of Sex Research, 25, 451-477.

Herek, G. \& González-Rivera, M. (2006). Attitudes towards homosexuality among U.S. residents of Mexican descent. The Journal of Sex Research, 43, 122-135.

Herrera, M. E., Mathiesen, M. O., Morales, M. G., Proust, P. M. \& Vergara, M. A. (2006). Actitud del adulto a cargo de la sala cuna hacia el género y su relación con la calidad del ambiente educativo. Estudios Pedagógicos, 32(1), 7-19.

Ideas (1997). Primera Encuesta de Tolerancia y No Discriminación. Santiago, Chile: Fundación Ideas.

Ideas (2001). Segunda Encuesta de Tolerancia y No Discriminación. Santiago, Chile: Fundación Ideas.

Ideas (2003). Tercera Encuesta de Tolerancia y No Discriminación. Santiago, Chile: Fundación Ideas.

Inglehart, R. \& Welzel, C. (2005). Modernization, cultural change, and democracy. The human development sequence. Cambridge, Inglaterra: Cambridge University Press.

Instituto Nacional de la Juventud (2007). Sexualidad juvenil y exposición a riesgo sanitario: condicionantes nuevos y permanentes. En Instituto Nacional de la Juventud, Quinta Encuesta Nacional de la Juventud (pp. 183-197). Santiago, Chile: Autor.

Maison, D., Greenwald, A. G. \& Bruin, R. H. (2004). Predictive validity of the Implicit Association Test in studies of brands, consumer attitudes, and behavior. Journal of Consumer Psychology, 14, 405-415.

Matus, C. (2005). El carrete como escenario. Una aproximación etnográfica a los códigos de la sexualidad ocasional en jóvenes urbanos. Última Década, 13(22), 9-37.

Merino, M. (2006). Propuesta metodológica de análisis crítico del discurso de la discriminación percibida. Revista Signos, 39, 453-469.

Mladinic, A., Saiz, J. L., Díaz, M., Ortega, A. \& Oyarce, P. (1998). Sexismo ambivalente en estudiantes universitarios chilenos: teoría, medición y diferencias de género. Revista de Psicología Social y Personalidad, 14, 1-14.

Montoya, G. J. (2006). Aproximación bioética a las terapias reparativas. Tratamiento para el cambio de la orientación sexual. Acta Bioethica, 12, 199-210.

Montoya-Montoya, G. J., Risco, L., Rodríguez, E., Valdebenito, C. \& Lolas, F. (2006). Confidencialidad de la orientación sexual en psiquiatría. Revista Chilena de Neuro-Psiquiatría, 44, 170-177.

Morrison, M. A. \& Morrison, T. G. (2002). Development and validation of a scale measuring modern prejudice toward gay men and lesbian women. Journal of Homosexuality, 43, 15-37.

Movimiento de Integración y Liberación Homosexual (2007). VI Informe Anual. Derechos humanos. Minorías sexuales chilenas. Hechos 2007. Santiago, Chile: Autor.

Nierman, A. J., Thompson, S. C., Bryan, A. \& Mahaffey, A. L. (2007). Gender roles beliefs and attitudes toward lesbians and gay men in Chile and the U.S. Sex Roles, 57, 61-67.

Nosek, B. A., Greenwald, A. G. \& Banaji, M. R. (2005). Understanding and using the Implicit Association Test: II. Method variables and construct validity. Personality and Social Psychology Bulletin, 31, 166-180.

Nosek, B. A., Greenwald, A. G. \& Banaji, M. R. (2007). The Implicit Association Test at age 7: A methodological and conceptual review. En J. A. Bargh (Ed.), Automatic processes in social thinking and behavior (pp. 265-292). Philadelphia, PA: Psychology Press. 
Ortiz-Hernández, L. (2004). La opresión de minorías sexuales desde la perspectiva de género. Política y Cultura, 22, 161182.

Ortiz-Hernández, L. \& García, M. I. (2005a). Opresión internalizada y prácticas sexuales de riesgo en varones homo-y bisexuales de México. Revista de Saúde Pública, 39, 956-964.

Ortiz-Hernández, L. \& García, M. I. (2005b). Efectos de la violencia y la discriminación en la salud mental de bisexuales, lesbianas y homosexuales de la Ciudad de México. Cadernos de Saúde Pública, 21, 913-925.

Petty, R. E. \& Wegener, D. T. (1998). Attitude change. En D. T. Gilbert, S. T. Fiske \& G. Lindzey (Eds.), The handbook of social psychology (4a ed., pp. 323-390). New York: McGrawHill.

Poehlman, T. A., Uhlmann, E., Greenwald, A. G. \& Banaji, M. R. (2006). Understanding and using the Implicit Association Test: III. Meta-analysis of predictive validity. Manuscrito no publicado, Yale University, New Heaven, CT, Estados Unidos.

Programa Conjunto de las Naciones Unidas sobre el VIH/Sida ONUSIDA (2006). Derechos humanos, saludy VIH. Guías de acciones estratégicas para prevenir y combatir la discrimina- ción por orientación sexual e identidad de género. Bogotá: Autor. Extraído el 15 Junio, 2008, de http://www.onusida.org. co/derechos\%20humanos\%20salud\%20y\%20VIH.pdf

Sevilla, M. L. \& Álvarez, N. E. (2006). Normalización del discurso homofóbico: aspectos bioéticos. Acta Bioethica, $12,211-217$.

Sharim, D. (2005). La identidad de género en tiempos de cambio: una aproximación desde los relatos de vida. Psykhe,14(2), 19-32.

Valenzuela, E. \& Casas, L. (2007). Derechos sexuales y reproductivos: confidencialidad y VIH en adolescentes chilenos. Acta Bioethica, 13, 207-215.

Vega, J., Bedregal, P., Jadue, L. \& Delgado, I. (2003). Equidad de género en el acceso a la atención de salud en Chile. Revista Médica de Chile, 131, 669-678.

World Values Survey (2005). World Values Survey. Stockholm, Suecia: Autor. Extraído el 15 Mayo, 2008, de http://www. worldvaluessurvey.org

Fecha de recepción: Marzo de 2008.

Fecha de aceptación: Septiembre de 2008. 
